UT-KOMABA/07-5

UT-07-13

\title{
Gauge Mediation with $D$-term SUSY Breaking
}

\author{
Y. Nakayama, ${ }^{1}$ Masato Taki ${ }^{2}{ }^{\text {Taizan }}$ Watari $^{2}$ and T. T. Yanagida ${ }^{2}$ \\ ${ }^{1}$ Institute of Physics, University of Tokyo, \\ Komaba, Meguro-ku, Tokyo 153-8902, Japan \\ ${ }^{2}$ Department of Physics, University of Tokyo, \\ Hongo, Bunkyo-ku, Tokyo 113-0033, Japan
}

\begin{abstract}
We construct a gauge-mediation model with a $D$-term supersymmetry (SUSY) breaking. $R$-symmetry breaking necessary for generating the SUSY standard-model gaugino masses is given by gaugino condensation of a strongly coupled gauge theory in the hidden sector. The energy scale of the strong dynamics of the hidden sector gauge theory should be around the messenger mass scale $M$, or otherwise perturbative calculations would be reliable and would lead to negative soft mass squared for squarks and sleptons. Thus, all the mass scales are controlled by a virtually single parameter, $\sqrt{D} / M$. This model covers a very wide range of gravitino mass, $m_{3 / 2} \simeq 1 \mathrm{eV}-100 \mathrm{TeV}$. Possible embeddings of the model in string theory are also discussed.
\end{abstract}




\section{Introduction}

Supersymmetry (SUSY) can be broken either in the $F$-term or in the $D$-term (or both), but realistic models of SUSY breaking have been discussed almost exclusively in the $F$-term SUSY breaking. Two major issues of the $D$-term SUSY breaking scenario are how to obtain $R$-symmetry breaking and how to avoid tachyonic masses (negative mass squared) for sfermions. Since a non-vanishing Fayet-Iliopoulos $D$-term parameter does not break an $R$-symmetry, something extra is necessary in order to obtain $R$-symmetry breaking gaugino masses in the SUSY standard-model (SSM) 11 Furthermore, in the gravity mediation with a generic Kähler potential, soft mass-squared parameters of scalar fields from supergravity scalar potential,

$$
V=e^{K / M_{P}^{2}}\left(|F|^{2}-3\left|W / M_{P}\right|^{2}\right)+D^{2} / 2 g^{2},
$$

are negative when the expectation value of $|W|^{2}$ is chosen so that the cosmological constant vanishes. Tachyonic squarks and sleptons imply that the color and electromagnetic U(1) symmetries would be broken in the vacuum.

Gauge group often becomes a product group, $G=G_{1} \times G_{2} \times \cdots$, in string theory compactification with D-branes. Only a part of the product gauge group is identified with $\mathrm{SU}(5)_{\mathrm{GUT}}$ or with the standard-model gauge group $\mathrm{SU}(3)_{C} \times \mathrm{SU}(2)_{L} \times \mathrm{U}(1)_{Y}$, and all other factors may become the hidden sector. When a $U(1)$ factor is contained in the gauge group, it may have a non-vanishing $D$-term expectation value. If one of the hidden sector gauge groups is strongly coupled, and gaugino condensation is formed, $R$-symmetry is broken. Therefore, the only problem is how to mediate such an $R$-symmetry breaking to the standard-model sector.

In this article, we construct a model of gauge mediation in the $D$-term SUSY breaking scenario 2 where the $R$-symmetry breaking from gaugino condensation is mediated through a messenger sector. The tachyonic mass problem may also be addressed in the gauge mediation because the tachyonic contribution from supergravity is small and negligible. We find the SUSY-breaking mass squared can be positive in our model of gauge mediation only when the hidden sector gauge group is strongly coupled around the energy scale of the masses of the messenger fields.

This phenomenological constraint fixes one of freedoms in choosing parameters of the model. The $D$-term SUSY breaking scenario has three important parameters, namely, SUSY-breaking scale $\sqrt{D} / g$, the messenger mass scale $M$ and the $R$-symmetry breaking

\footnotetext{
${ }^{1}$ For models with gaugino masses that preserve $\mathrm{U}(1)_{R}$ symmetry, see e.g. [1].

${ }^{2}$ See [2] and references therein for another line of models with $D$-term gauge mediation.
} 
scale $\Lambda$. However, $\Lambda$ should be close to $M$ to avoid the negative mass squared for squarks and sleptons, but $\sqrt{D}$ and $M$ are chosen freely. This situation is similar to gauge mediation in the $F$-term SUSY-breaking scenario [3], where $\sqrt{F}$ and $M$ are free. Depending on the value of $\sqrt{D} / M$, gravitino mass ranges from about $1 \mathrm{eV}$ to $100 \mathrm{TeV}$ in our mediation model in the $D$-term SUSY breaking scenario. The gravitino mass can be that light because the SUSY breaking in a $D$-term of a U(1) symmetry is directly mediated to the visible sector through messenger fields charged under the U(1) symmetry. Light gravitino mass is very attractive from a phenomenological point of view.

We also discuss possible embeddings of the gauge mediation model in string theory. Fractional D3-branes located at a conifold singularity of a Calabi-Yau three-fold [4] constitute a hidden sector while the standard-model sector is assumed to be realized on D7-branes on a four-cycle of the three-fold. D3-D7 strings become the messenger sector. Blow-up of the two-cycle at the conifold singularity breaks SUSY [5] with a non-zero $D$-term.

This paper is organized as follows. In section 2, we present a field theory model of $D$-term gauge mediation. This phenomenological model is embedded in string theory in section 3. Conclusions and discussions are found in section 4. In appendix A, we summarize the details of the two-loop computation for the SUSY scalar mass squared in the $D$-term gauge mediation. In appendix $\mathrm{B}$, more examples of string theory embeddings can be found.

\section{Gauge mediation with $D$-term SUSY breaking}

In the $D$-term SUSY-breaking scenario, we have a U(1) gauge symmetry, whose $D$-term expectation value $\langle D\rangle=g^{2} \xi$ breaks SUSY. This $\mathrm{U}(1)$ symmetry is referred to as $\mathrm{U}(1)_{D}$ symmetry. The $D$-term expectation value itself, however, does not break $R$-symmetry, while any $R$-symmetries have to be broken down to $\mathbb{Z}_{2}$ so that Majorana gaugino masses are allowed. Thus, we introduce an $\mathrm{SU}(N)$ super Yang-Mills multiplet, which leads to its gaugino condensation at low-energy:

$$
\frac{1}{32 \pi^{2}}\left\langle 2 \operatorname{tr}_{N}\left(\lambda^{\alpha} \lambda_{\alpha}\right)\right\rangle=\Lambda_{N}^{3}
$$

where $\Lambda_{N}$ is the dynamical scale of the $\mathrm{SU}(N)$ gauge group. Here and hereafter, $\operatorname{tr}_{N}$ denotes the trace over the fundamental representation of this $\mathrm{SU}(N)$ gauge group. The gaugino condensation breaks $R$-symmetry down to $R$-parity. This is probably the minimum construction of the hidden sector of the $D$-term SUSY breaking scenario. 




(a)

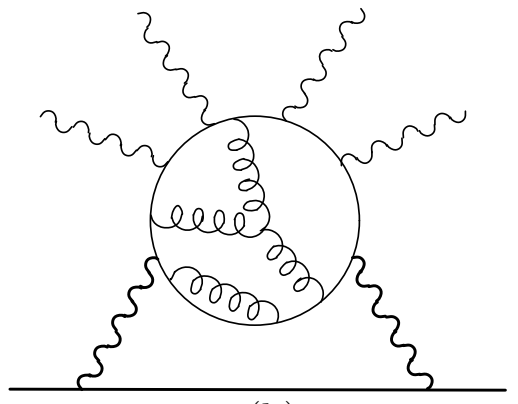

(b)

Figure 1: Supergraphs contributing to squarks and slepton masses. Chiral multiplets in the visible sector are denoted by straight solid lines at the bottom, and the messenger chiral multiplets are the running in the loop. Gluon-like lines are used for the $\mathrm{SU}(N)$ vector multiplet, thick photon-like ones for the $\mathrm{SU}(5)_{\mathrm{GUT}}$ and ordinary photon-like ones for $\mathrm{U}(1)_{D}$.

In order for the SUSY breaking in the hidden sector to be mediated to the visible particle-physics sector, messenger chiral multiplets $\psi$ and $\bar{\psi}$ are introduced. They are in the $\mathbf{5}$ and $\overline{\mathbf{5}}$ representations, respectively, of the $\mathrm{SU}(5)_{\text {GUT }}$ symmetry containing the standard-model gauge group $\mathrm{SU}(3)_{C} \times \mathrm{SU}(2)_{L} \times \mathrm{U}(1)_{Y}$. If the messenger chiral multiplets carry non-vanishing charges of the $\mathrm{U}(1)_{D}$ symmetry, the mass matrix of the complex scalars is of the form

$$
V \sim\left(\begin{array}{cc}
\psi^{\dagger} & \bar{\psi}^{T}
\end{array}\right)\left(\begin{array}{cc}
M^{2}+g^{2} \xi & 0 \\
0 & M^{2}-g^{2} \xi
\end{array}\right)\left(\begin{array}{c}
\psi \\
\bar{\psi}^{*}
\end{array}\right)
$$

in contrast to a familiar form in gauge-mediation models in the F-term SUSY breaking scenario,

$$
V \sim\left(\begin{array}{cc}
\psi^{\dagger} & \bar{\psi}^{T}
\end{array}\right)\left(\begin{array}{cc}
M^{2} & F \\
F^{*} & M^{2}
\end{array}\right)\left(\begin{array}{c}
\psi \\
\bar{\psi}^{*}
\end{array}\right)
$$

Here, $M$ is the SUSY-invariant mass of the messenger multiplets, $W=M \psi \bar{\psi}$ (We choose $M$ to be real). It has to be large enough, $M^{2}>|\langle D\rangle|$, or otherwise $\psi$ (or $\bar{\psi}$ ) would develop an expectation value, breaking the symmetry of the standard model. In order for the $R$ symmetry breaking to be mediated as well, we assume that the messenger multiplets $\psi$ and $\bar{\psi}$ are in the $(N,+1,5)$ and $(\bar{N},-1, \overline{\mathbf{5}})$ representations of the $\mathrm{SU}(N) \times \mathrm{U}(1)_{D} \times \mathrm{SU}(5)_{\mathrm{GUT}}$ gauge group.

Soft SUSY-breaking mass squared is generated for scalar particles in the visible sector after integrating out messenger fields. Two-loop diagrams in Figure 1(a) induce an effective operator in the Kähler potential, 


$$
K_{\mathrm{eff}}=c \frac{g_{\mathrm{GUT}}^{4}}{16 \pi^{2}} \frac{1}{16 \pi^{2}} \frac{\left|\mathcal{W}^{\alpha} \mathcal{W}_{\alpha}\right|^{2}}{M^{6}} f_{i} f_{i}^{\dagger}
$$

where $c$ stands for coefficients of order unity and $g_{\mathrm{GUT}}$ for the gauge coupling constants of the SSM. $f_{i}$ are matter chiral multiplets in the visible sector and $\mathcal{W}^{\alpha}=\lambda^{\alpha}+\theta^{\alpha} D+\cdots$ the field strength superfield for the $\mathrm{U}(1)_{D}$ vector multiplet 3 Replacing the $\mathrm{U}(1)_{D}$ superfield $\mathcal{W}^{\alpha}$ by its SUSY-breaking expectation value $\theta^{\alpha}\langle D\rangle$, we obtain soft masses squared of order

$$
m_{i}^{2} \sim\left[\frac{\alpha_{\mathrm{GUT}}}{4 \pi} \frac{\langle D\rangle^{2}}{M^{3}}\right]^{2} .
$$

Note that the soft mass squared is not generated at the order of $(\langle D\rangle / M)^{2}$, as opposed to the $F$-term SUSY breaking scenario. Perturbative 2-loop calculation, whose details are found in appendix A, confirms that all the contributions of order $(\langle D\rangle / M)^{2}$ cancel out. The leading contribution of order (6) turns out to be negative, as seen in appendix A. The first couple of terms in $|\langle D\rangle| / M^{2}$ expansion are all negative, and one can further see by evaluating (17) numerically that all the squarks and sleptons have tachyonic masses as long as $M^{2}>|\langle D\rangle|$.

The perturbative calculation in appendix $\mathrm{A}$, however, is not reliable if the $\mathrm{SU}(N)$ gauge theory in the hidden sector is strongly coupled around the threshold of the messenger fields. The $\mathrm{SU}(N)$ gauge theory turns from a five-flavor SUSY "QCD" to a pure super Yang-Mills theory at the threshold, and its gauge coupling constant becomes strong immediately below the threshold for sufficiently large $N$. Soft masses squared from all sorts of diagrams in Figure 1 (b) are of the same order as (6); higher loop amplitudes are not suppressed because the loop factors $\left(g_{N}^{2} N / 16 \pi^{2}\right)^{n}$ are of order unity, and effective operators with higher power of $\left|\operatorname{tr}_{N}\left(\mathcal{W}^{\alpha} \mathcal{W}_{\alpha}\right)\right| / M^{3}$ are just as important as the leading order operator (5) because

$$
\left\langle 2 \operatorname{tr}_{N}\left(\mathcal{W}^{\alpha} \mathcal{W}_{\alpha}\right)\right\rangle=32 \pi^{2} \Lambda_{N}^{3} \approx M^{3}
$$

when the $\mathrm{SU}(N)$ interactions are strong just below the threshold 4 Since it is impossible

\footnotetext{
${ }^{3}$ We use a convention so that gauge kinetic terms are $\int d^{2} \theta\left[\frac{1}{4 g^{2}} \mathcal{W}^{\alpha} \mathcal{W}_{\alpha}+\frac{1}{2 g^{2}} \operatorname{tr}_{N} \mathcal{W}^{\alpha} \mathcal{W}_{\alpha}\right]+$ h.c..

${ }^{4}$ The dynamical scale $\Lambda$ of the $\mathrm{SU}(N)$ gauge theory cannot be chosen above the messenger mass scale for $N=2,3$. This is because the $\mathrm{SU}(N)$ gauge theory is in the conformal window. For $N=4,5, \Lambda$ may well, in principle, be much larger than the messenger mass scale $M$. A naive estimate of the leading contribution to soft mass-squared of squarks and sleptons is negative for $N=4$, but the leading calculable contribution of order $\left(\alpha_{\mathrm{GUT}} / 4 \pi\right)^{2} D^{4} / M^{6}$ vanishes for $N=5$, and the sign of leading order non-vanishing contrbution, which is of order $\left(\alpha_{\mathrm{GUT}} / 4 \pi\right)^{2} D^{4} / \Lambda^{6}$, is not calculable. Thus, it is not clear in the $M \ll \Lambda$ limit, whether the SUSY-breaking mass-sqared of squarks and sleptons are negative or not. $N \geq 6$ is not compatible with perturbative unification and low-scale gauge mediation, and hence of less theoretical interest.
} 

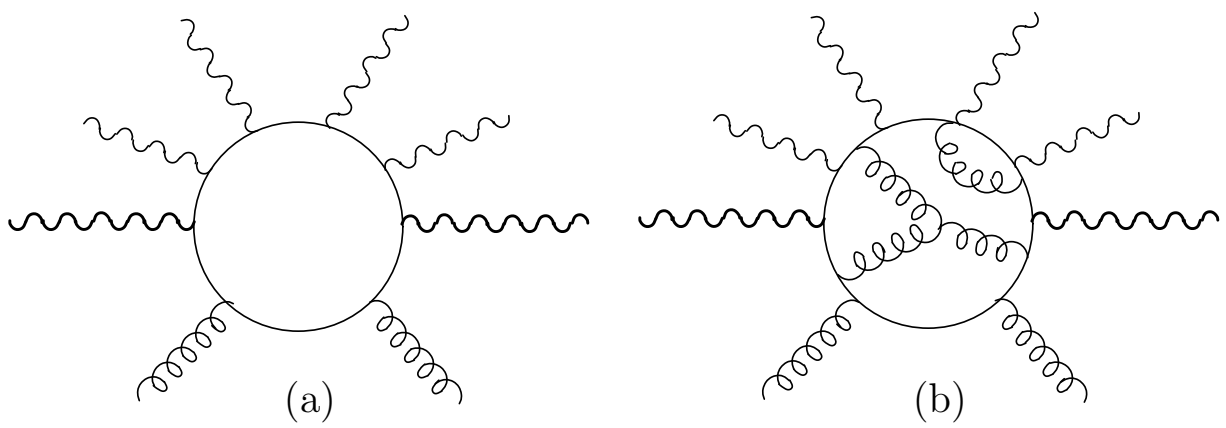

Figure 2: Supergraphs contributing to gaugino masses. See the caption of Figure 1 for the meaning of various lines.

to calculate all those contributions in such a strong-coupling regime, there is practically no way to know whether or not squarks and sleptons have tachyonic masses. Thus, we just assume that the sign is positive for all squarks and sleptons 5 Because of the naive-dimensional-analysis argument [17], eq. (6) still gives a reliable estimate of the soft SUSY-breaking masses squared even in this strong-coupling regime.

Gaugino masses of the standard-model gauge groups originate from one-loop diagrams of messengers in Figure2(a). All diagrams in Figure2(b), which are dressed by the $\mathrm{SU}(N)$ interactions also contribute to the gaugino masses by the same order of magnitude. Those diagrams induce an effective operator in the Kähler potential

$$
K_{\text {eff }}=c^{\prime} \frac{1}{16 \pi^{2}} \frac{\left|\mathcal{W}^{\alpha} \mathcal{W}_{\alpha}\right|^{2}}{M^{10}} \operatorname{tr}_{N}\left(\mathcal{W}^{\alpha \dagger} \mathcal{W}_{\alpha}^{\dagger}\right) \operatorname{tr}_{\mathrm{GUT}}\left(\mathcal{W}^{\alpha} \mathcal{W}_{\alpha}\right)+\text { h.c. }
$$

with a coefficient $c^{\prime}$ of order unity. Field-strength superfields in $\operatorname{tr}_{\text {GUT }}$ are those of the SSM. Thus, the gaugino masses of the SSM are of order

$$
m_{\text {gaugino }} \simeq \frac{\alpha_{\mathrm{GUT}}}{4 \pi} \frac{32 \pi^{2} \Lambda_{N}^{3}}{M^{3}} \frac{\langle D\rangle^{4}}{M^{7}},
$$

where we have replaced $\operatorname{tr}_{N}\left(\mathcal{W}^{\dagger} \mathcal{W}^{\dagger}\right)$ by its expectation value.

Assuming that the $\mathrm{SU}(N)$ interactions are strongly coupled around the threshold, and hence (7), we see that the second factor $\left(32 \pi^{2} \Lambda_{N}^{3} / M^{3}\right)$ does not suppress gaugino masses relatively to sfermion masses.

Although the $D$-term SUSY breaking scenario at first seems to involve two independent parameters $\Lambda / M$ and $\sqrt{D} / M$, the former cannot be chosen freely for realistic models without tachyonic masses for squarks or sleptons. Therefore, the spectrum predicted

\footnotetext{
${ }^{5}$ See [6, 7] for a similar argument for the strongly coupled $F$-term gauge mediation.
} 
from the present model depends only on one parameter, $\sqrt{D} / M$. As we have already seen, SUSY-breaking parameters in the visible sector,

$$
m_{\tilde{g}, \tilde{w}, \tilde{b}} \approx \frac{\alpha_{\mathrm{GUT}}}{4 \pi} \frac{\langle D\rangle^{4}}{M^{7}}, \quad m_{\tilde{q}, \tilde{l}} \approx \frac{\alpha_{\mathrm{GUT}}}{4 \pi} \frac{\langle D\rangle^{2}}{M^{3}},
$$

depend on $\sqrt{D} / M$ in an interesting way.

The largest possible $\sqrt{D} / M$ corresponds to the lightest gravitino mass possible. Messenger fields do not have tachyonic masses while $\sqrt{D} / M \lesssim 1$, and at this limit, sfermion masses and gaugino masses are comparable (and both are supposed to be around the electroweak scale), and

$$
M \sim \sqrt{D} \sim 100 \mathrm{TeV}, \quad m_{3 / 2} \sim \mathcal{O}(\mathrm{eV}) .
$$

Theories with such a light gravitino is free from the constraint on the relic density of hot dark matter 6 Reheating temperature can be arbitrarily high, and in particular, thermal leptogenesis is not constrained in any ways by cosmological problems associated with gravitino.

If the SUSY breaking scale $\sqrt{D}$ is not as high as the messenger scale $M$, on the other hand, sfermions are much heavier than gauginos:

$$
m_{\text {sfermion }} \sim\left(\frac{M}{\sqrt{D}}\right)^{4} \times m_{\text {gaugino }},
$$

and the messenger scale and SUSY-breaking scale are

$$
M \sim\left(\frac{M}{\sqrt{D}}\right)^{8} \times 100 \mathrm{GeV}, \quad \sqrt{D} \sim\left(\frac{M}{\sqrt{D}}\right)^{7} \times 100 \mathrm{GeV}, \quad m_{3 / 2} \sim\left(\frac{M}{\sqrt{D}}\right)^{14} \times \mathcal{O}(\mathrm{eV}) .
$$

for $m_{\text {gaugino }} \simeq 100 \mathrm{GeV}$. The anomaly mediated contributions to gaugino masses are negligible for $\sqrt{D} / M \lesssim 1 / 10$ because the gravitino mass is no more than about $100 \mathrm{TeV}$. The spectrum of split SUSY [8] is realized without assuming a particular form of Kähler potential.

The size of the hidden-sector gauge group $N$ is arbitrary except that there should not be too much messenger fields. The messenger fields add $N$ pairs of chiral multiplets in the $\mathrm{SU}(5)_{\mathrm{GUT}}-\mathbf{5}+\overline{\mathbf{5}}$ representations. The gauge couplings of the standard-model gauge groups become asymptotic non-free, and may no longer be perturbative below the scale of gauge coupling unification. Since the gauge coupling unification is one of the most important motivations for low-energy SUSY, we do not want to lose it. If the messenger mass scale is of order $100 \mathrm{TeV}$, then the standard-model gauge couplings remain perturbative for $N \leq 6$. For the messenger mass scale of order $10^{12} \mathrm{GeV}, N \lesssim 20$.

\footnotetext{
${ }^{6}$ The dominant dark matter may be axion or some other (possibly hidden) particle.
} 


\section{$3 \quad D$-term SUSY breaking models in string theory}

It is rather straightforward to realize the idea of the $D$-term gauge mediation in string theory. Let us consider a Calabi-Yau orientifold compactification of the Type IIB string theory. The standard-model gauge groups arise from a stack of five D7-branes wrapped on a holomorphic four-cycle $\Sigma$ in a Calabi-Yau manifold $X$. Quarks, leptons and Higgs super multiplets may arise from D7-D7 intersection. It is certainly a technically involved issue to find out an explicit geometry where exactly three generations of the matter multiplets are obtained, but we just assume in this article that there is such a geometry because we focus on parts of geometry that is essential to a gauge mediation.

Suppose that the Calabi-Yau three-fold $X$ has a conifold singularity that is not contained in the four-cycle $\Sigma$ where the standard-model gauge fields propagate. When $N$ fractional D3-branes are at the singularity, a $\mathrm{U}(N)$ super Yang-Mills theory of $\mathcal{N}=1$ SUSY is on the D3-branes 7 The $\mathrm{SU}(N)$ part can be identified with the hidden-sector $\mathrm{SU}(N)$, whose gaugino condensation breaks $R$-symmetry down to $R$-parity.

SUSY can be broken if $S^{2}$ at the conifold singularity is blown up. The SUSY-breaking vacuum energy is described in the effective field theory language as a non-vanishing FayetIliopoulos parameter of the U(1) part vector multiplet. Thus, the U(1) part and its Fayet-Iliopoulos parameter can be identified with what we have needed in the $D$-term SUSY breaking model in section 2. In the string compactification with the finite internal volume, the Fayet-Iliopoulos parameter becomes a dynamical moduli. Once the Kähler moduli responsible for the Fayet-Iliopoulos parameter is dynamical, SUSY is restored by relaxing it down to $\xi=0$. We assume that the resolution Kähler moduli responsible for $\xi$ is fixed by some other mechanisms 8

Open strings connecting those fractional D3-branes and the five D7-branes wrapped on the four-cycle $\Sigma$ yield massive particles; their masses are proportional to the shortest distance between the blown-up $S^{2}$ and $\Sigma$. They are in the vector-like pair of representations, $(N,+1,5)$ and $(\bar{N},-1, \overline{\mathbf{5}})$, just as in section 2 . Those particles are identified with the messenger sector chiral multiplets, $\psi$ and $\bar{\psi}$. By introducing fractional D3-branes at a conifold singularity, we have exactly what we need in the phenomenological model in section 2. Since the low-energy effective theory of this Type IIB compactification is exactly the same as the field-theory model in section 2 , all the results discussed in section 2 follow from this compactification.

\footnotetext{
${ }^{7}$ Generalizations to the case with regular D3-branes are presented in appendix B.

${ }^{8}$ Local geometry has to be made more complicated for this to happen. c.f. [9].
} 


\section{Discussions and Conclusion}

In this paper, we have proposed a new gauge mediation scenario based on the $D$-term SUSY breaking in the hidden sector. A crucial ingredient of our scenario is the gaugino condensation in the hidden sector, which yields the necessary $R$-symmetry breaking to produce the SSM gaugino mass. Notice that there is no $R$-axion problem [10] because the $R$-symmetry is anomalous in our model.

Unfortunately, the perturbative two-loop computation shows that the SUSY scalar particles have negative masses-squared. Therefore, to apply the $D$-term gauge mediation successfully to our real world, the hidden sector should be strongly coupled near the thresholds of messengers. This requirement reduces the number of parameters of our model and makes our prediction even more concrete. Furthermore, this is rather a natural assumption because the hidden $\mathrm{SU}(N)$ gauge interactions become more asymptotic free after decoupling of the messenger fields. A possible explanation of the origin of the strong coupling at the threshold may be approximate conformal invariance above the messenger [7.

Note that this $D$-term breaking scenario has virtually only one gaugino-to-sfermion mass ratio and the gravitino mass. For the largest $\sqrt{D} / M \sim 1$, sleptons are lighter than gauginos and the gravitino mass is of order eV. For a smaller value of $\sqrt{D} / M$, sfermions are much heavier than gauginos, like in split SUSY, but the masses of those particles come from gauge mediation.

We have also discussed an embedding of $D$-term gauge mediation into the type IIB superstring theory. We have shown that the fractional D3-branes at the (resolved) conifold singularity together with the standard model flavor D7-branes realize the simplest $D$-term gauge mediation, given that the Fayet-Iliopoulos parameter is fixed. Generalized models presented in appendix B possess the gravity dual description. It would be interesting if we could find any evidence, from the supergravity viewpoint, for the positivity of the SUSY scalar mass-squared for squarks and sleptons, while strongly coupled nature of the $\mathrm{SU}(N)$ theory hinders the direct field theory computation.

\section{Acknowledgements}

Y.N. thanks the Japan Society for the Promotion of Science for financial support. 


\section{A Two-loop soft scalar mass squared from D-term SUSY breaking}

We report the two-loop soft scalar mass squared from gauge mediation with $D$-term SUSY

breaking in this appendix. Under the $D$-term SUSY breaking, the mass squared for the messenger scalar takes the form as

$$
m^{2}=\left(\begin{array}{cc}
m_{+}^{2} & 0 \\
0 & m_{-}^{2}
\end{array}\right)=\left(\begin{array}{cc}
m_{f}^{2}+D & 0 \\
0 & m_{f}^{2}-D
\end{array}\right)
$$

where $m_{f}=M$ is the mass for the fermion.

Following the notation of [11], we find that the mass squared for the scalar SUSY particle is given by

$$
\begin{aligned}
\frac{2\left(16 \pi^{2}\right)^{2}}{g^{4}} m_{0}^{2} & =-2\left\langle m_{+}\left|m_{+}\right| 0\right\rangle-2\left\langle m_{-}\left|m_{-}\right| 0\right\rangle-4 m_{+}^{2}\left\langle m_{+}\left|m_{+}\right| 0,0\right\rangle-4 m_{-}^{2}\left\langle m_{-}\left|m_{-}\right| 0,0\right\rangle \\
& -4\left\langle m_{f}\left|m_{f}\right| 0\right\rangle+8 m_{f}^{2}\left\langle m_{f}\left|m_{f}\right| 0,0\right\rangle+4\left\langle m_{+}\left|m_{f}\right| 0\right\rangle+4\left\langle m_{-}\left|m_{f}\right| 0\right\rangle \\
& +4\left(m_{+}^{2}-m_{f}^{2}\right)\left\langle m_{+}\left|m_{f}\right| 0,0\right\rangle+4\left(m_{-}^{2}-m_{f}^{2}\right)\left\langle m_{-}\left|m_{f}\right| 0,0\right\rangle
\end{aligned}
$$

where

$$
\begin{aligned}
\left\langle m_{1}\left|m_{2}\right| m_{3}\right\rangle & =\int \frac{d^{4} q}{(2 \pi)^{4}} \frac{d^{4} k}{(2 \pi)^{4}} \frac{1}{\left(q^{2}+m_{1}^{2}\right)\left(k^{2}+m_{2}^{2}\right)\left([k-q]^{2}+m_{3}^{2}\right)} \\
\left\langle m_{1}\left|m_{2}\right| m_{3}, m_{3}\right\rangle & =\int \frac{d^{4} q}{(2 \pi)^{4}} \frac{d^{4} k}{(2 \pi)^{4}} \frac{1}{\left(q^{2}+m_{1}^{2}\right)\left(k^{2}+m_{2}^{2}\right)\left([k-q]^{2}+m_{3}^{2}\right)^{2}}
\end{aligned}
$$

After some algebra, we finally obtain

$$
\begin{aligned}
\frac{2\left(16 \pi^{2}\right)^{2}}{g^{4}} m_{0}^{2} & =4\left[\left(4 M^{2}\right) \log \left(M^{2}\right)^{2}+\log \left(M^{2}-D\right)\left(2\left(M^{2}-D\right)+\left(2 M^{2}-D\right) \log \left(M^{2}-D\right)\right)\right. \\
& +\log \left(M^{2}+D\right)\left(2\left(M^{2}+D\right)+\left(2 M^{2}+D\right) \log \left(M^{2}+D\right)\right) \\
& -2 \log \left(M^{2}\right)\left(2 M^{2}+\left(2 M^{2}-D\right) \log \left(M^{2}-D\right)+\left(2 M^{2}+D\right) \log \left(M^{2}+D\right)\right) \\
& +2 D \operatorname{Li}_{2}\left(\frac{D}{D-M^{2}}\right)+\left(D+2 M^{2}\right) \operatorname{Li}_{2}\left(\frac{D^{2}}{M^{4}}\right) \\
& \left.-2 D\left(2 \operatorname{Li}_{2}\left(\frac{D}{M^{2}}\right)+\operatorname{Li}_{2}\left(\frac{D}{D+M^{2}}\right)\right)\right]
\end{aligned}
$$


where $\operatorname{Li}_{2}(x)=\sum_{k=1}^{\infty} \frac{x^{k}}{k^{2}}$ is the dilogarithm function. This expression is a special case of eqs. (2.6-2.8) of [18]. By expanding (17) with respect to $D / M^{2}$, we obtain

$$
\frac{2\left(16 \pi^{2}\right)^{2}}{g^{4}} m_{0}^{2}=-\frac{14 D^{4}}{9 M^{6}}-\frac{76 D^{6}}{75 M^{10}}-\frac{341 D^{8}}{490 M^{14}}-\cdots \text {. }
$$

Note that the leading term is not $O\left(D^{2} / M^{2}\right)$ but $O\left(D^{4} / M^{6}\right)$ consistent with the effective operator analysis. The first three terms of the soft mass squared in (18) are all negative, and one can even see numerically that (17) is negative for any $M^{2}>|\langle D\rangle|$.

\section{B Generalized models in string theory}

The hidden sector of the model in section 3 consists of $N$ fractional D3-branes on local (resolved) conifold singularity. This hidden sector can be extended to a system of $N+p$ fractional D3-branes and $p$ fractional D3-branes of the other type placed at the (resolved) conifold singularity. The gauge group of the hidden sector is $\mathrm{SU}(N+p) \times \mathrm{SU}(p) \times$ $\mathrm{U}(1)_{B} \times \mathrm{U}(1)_{C M}$; both the $\mathrm{U}(1)_{B}$ and $\mathrm{U}(1)_{C M}$ symmetries are gauged because we consider a compact manifold $X 9$ The matter contents of the hidden and messenger sectors are summarized in Table 1. This D-brane system is equivalent to $N$ D5-branes wrapped on the vanishing two-cycle at the conifold singularity and $p$ ordinary D3-branes. The model in section 3 corresponds to the special case $p=0$, and hence this class of models are generalization of the model in section 3 ,

The $\mathrm{U}(1)_{B}$ symmetry may have a non-vanishing Fayet-Iliopoulos parameter $\xi_{B}$ [5]. From the string theory perspective, it corresponds to blowing up a vanishing $S^{2}$ cycle at the conifold singularity. Once the moduli are fixed, then we know that this hidden-sector gauge theory breaks SUSY unless $p$ is an integral multiple of $N$ [5].

\section{Vacuum Moduli in the Hidden Sector}

Matter fields in the hidden sector consists of four chiral multiplets, $A_{\alpha}(\alpha=1,2)$ and $B_{\dot{\alpha}}(\dot{\alpha}=1,2)$ as in table 1. They have a tree-level superpotential

$$
W_{\text {tree }}=\frac{1}{\mu}\left(A_{1} B_{1} A_{2} B_{2}-A_{1} B_{2} A_{2} B_{1}\right) .
$$

The hidden sector gauge theory (for $\xi_{B}=0$ ) has a vacuum moduli space. In a simplest case, $N=2$ and $p=1$, for example, the quantum vacuum moduli is parametrized by

\footnotetext{
${ }^{9}$ In fact, whether the vector fields of those $U(1)$ symmetries remain massless depends on the details of the full compactification (see e.g. [12] for a recent discussion). Introduction of fluxes and the moduli stabilization make the problem even more complicated.
} 


\begin{tabular}{c|cccccc} 
& $\mathrm{SU}(N+p)$ & $\mathrm{SU}(p)$ & $\mathrm{U}(1)_{B}$ & $\mathrm{U}(1)_{C M}$ & $\mathrm{SU}(5)_{\mathrm{GUT}}$ & {$[\mathrm{SU}(2) \times \mathrm{SU}(2)]_{F}$} \\
\hline$A_{\alpha}$ & $N+p$ & $\bar{p}$ & +2 & 0 & & $2 \times 1$ \\
$B_{\dot{\alpha}}$ & $\overline{N+p}$ & $p$ & -2 & 0 & & $1 \times 2$ \\
\hline$\psi$ & $N+p$ & & +1 & +1 & $\mathbf{5}$ & - \\
$\tilde{\psi}$ & $\overline{N+p}$ & & -1 & -1 & $\overline{\mathbf{5}}$ & - \\
$\Psi$ & & $p$ & -1 & +1 & $\mathbf{5}$ & - \\
$\tilde{\Psi}$ & & $\bar{p}$ & +1 & -1 & $\overline{\mathbf{5}}$ & -
\end{tabular}

Table 1: The matter contents of the SUSY breaking sector and messengers. Inclusion of messengers breaks the flavor $[\mathrm{SU}(2) \times \mathrm{SU}(2)]_{F}$ symmetry.

meson superfields $\mathcal{M}_{\alpha \dot{\alpha}} \sim A_{\alpha} B_{\dot{\alpha}}$ with a constraint

$$
\mathcal{M}_{11} \mathcal{M}_{22}-\mathcal{M}_{12} \mathcal{M}_{21}= \pm \sqrt{\Lambda_{3}^{7} \mu}
$$

which is the defining equation of (two copies of) the deformed conifold [4, 5]. Here, the dynamical scale $\Lambda_{3}$ is defined by

$$
\Lambda_{3}^{7}=M^{7} e^{-\frac{2 \pi}{\alpha_{3}(M)}}
$$

with the use of the gauge coupling constant $\alpha_{N+p}$ of $\mathrm{SU}(N+p)$ renormalized at the messenger mass scale $M$. We notice that the $S U(3)$ gauge group shows a gaugino condensation

$$
\frac{1}{32 \pi^{2}}\left\langle 2 \operatorname{tr}_{3}\left(\lambda^{\alpha} \lambda_{\alpha}\right)\right\rangle= \pm \sqrt{\frac{\Lambda_{3}^{7}}{\mu}} .
$$

This moduli space corresponds to where the $p=1$ D3-brane is located in a deformed conifold defined by the equation (20). The non-vanishing Fayet-Iliopoulos parameter of the $\mathrm{U}(1)_{B}$ symmetry cannot be absorbed because all those meson fields $\mathcal{M}_{\alpha \dot{\alpha}} \sim A_{\alpha} B_{\dot{\alpha}}$ are neutral under the baryonic $\mathrm{U}(1)_{B}$ symmetry. The vacuum energy, to leading order, is given by

$$
V=\frac{g_{B}^{2}}{2} \xi_{B}^{2}
$$

\section{Potential along the flat direction}

The leading order analysis shows a degeneracy of the non-supersymmetric vacua along the flat directions (20), but there is no reason to prevent the emergence of the potential along the radial direction of the deformed conifold after the breaking of the SUSY. To see the strongly coupled effects, we will study the gravity dual description. In the gravity description, the model with the non-zero Fayet-Iliopoulos parameter is represented by the so-called "warped-resolved-deformed conifold" [13, 5]. 
The pseudo-flat direction is then described by the probe D3-brane along the "warpedresolved-deformed conifold." The potential for such a probe brane comes from the nontrivial dilaton in the "warped-resolved-deformed conifold" [13, 5].

$$
V(t)=T_{3} H^{-1}(t)\left(e^{-\phi(t)}-1\right)=\frac{T_{3}}{\gamma} \frac{U^{2}}{e^{-\phi(t)}+1}
$$

in terms of the dilaton $\phi(t)$ and the warp factor $H(t)$, where $t$ denotes the radial direction of the "warped-resolved-deformed conifold". $U$ and $\gamma$ are related to the resolution/deformation parameter of the conifold respectively. Here we have also introduced the D3-brane tension $T_{3}$. The D3-brane is attracted toward $t=0$ and for large $t$, we have a very flat potential consistent with the field theory analysis. We note that the SUSY is broken even at $t=0$. For later purposes, let us study the potential value at $t=0$ and $t \rightarrow \infty$ more carefully. The dilaton here is normalized so that $\phi(\infty)=0$ and the potential there is $V(t \rightarrow \infty) \sim \frac{1}{2} g_{B}^{2} \xi_{B}^{2}$. On the other hand, for large $U, e^{-\phi(0)} \sim|U|^{3 / 4}$ [5]. Since $U^{2}=\left(\frac{\mu}{\Lambda_{3}}\right)^{2 / 3} \frac{\xi_{B}^{2}}{\Lambda_{3}^{4}}$ is typically large, the potential barrier is steep.

\section{Inclusion of messengers}

The scalar potential along the flat direction discussed so far is modified as the probe $p=1$ D3-brane approaches the four-cycle $\Sigma$ where the $\mathrm{SU}(5)_{\text {GUT }}$ gauge fields propagate. Let the four-cycle be defined locally in the original conifold by [14] 10

$$
\mathcal{M}_{11}=M^{2} \text {. }
$$

Fields in the hidden sector and messengers have a superpotential interaction [14]

$$
W=h \tilde{\psi} A_{1} \Psi+g \psi B_{1} \tilde{\Psi}+M_{1} \psi \tilde{\psi}+M_{2} \Psi \tilde{\Psi} .
$$

The parameters in the superpotential satisfy $M_{1} M_{2}=h g M^{2}$. As the probe D3-brane approaches the four-cycle, $\mathcal{M}_{11} \sim A_{1} B_{1} \sim M^{2}$, some fields in the messenger sector-D3D7 open strings - become massless. When $\left\langle A_{\alpha=1}^{a=3}\right\rangle=\left\langle B_{a=3, \dot{\alpha}=1}\right\rangle \sim M$, the mass matrix in

$$
W=\left(\tilde{\psi}_{a=3}, \tilde{\Psi}\right)\left(\begin{array}{cc}
M_{1} & h\left\langle A_{\alpha=1}^{a=3}\right\rangle \\
g\left\langle B_{a=3, \dot{\alpha}=1}\right\rangle & M_{2}
\end{array}\right)\left(\begin{array}{c}
\psi^{a=3} \\
\Psi
\end{array}\right)+M_{1}\left(\tilde{\psi}^{a=1,2} \psi_{a=1,2}\right)
$$

has a reduced rank.

\footnotetext{
${ }^{10}$ In the string compactification with the finite internal volume, $M^{2}$ would be an open-string moduli. We again assume that the moduli $M^{2}$ is fixed by some other mechanisms.
} 
Let the massless direction be

$$
\psi_{-}=\cos \theta \psi^{a=3}+\sin \theta \Psi, \quad \tilde{\psi}_{-}=\cos \theta \tilde{\psi}^{a=3}+\sin \theta \tilde{\Psi},
$$

We use a common mixing angle $\theta$ for $\psi_{-}$and $\tilde{\psi}_{-}$because $h=g$ at all order in perturbation theory, 11 and the $\mathrm{SU}(3) D$-term condition ensures that $\left\langle A_{1}^{a=3}\right\rangle=\left\langle B_{a=3, \dot{\alpha}=1}\right\rangle$. The $D$-term potential of the $\mathrm{U}(1)_{B}$ and $\mathrm{U}(1)_{C M}$ symmetries are

$$
V_{B}+V_{C M}=\frac{g_{B}^{2}}{2}\left(\xi_{B}+\cos (2 \theta)\left(\left|\psi_{-}\right|^{2}-\left|\tilde{\psi}_{-}\right|^{2}\right)\right)^{2}+\frac{g_{C M}^{2}}{2}\left(\left|\psi_{-}\right|^{2}-\left|\tilde{\psi}_{-}\right|^{2}\right)^{2} .
$$

The Fayet-Iliopoulos parameter $\xi_{B}$ induces negative mass squared for either $\psi_{-}$or $\tilde{\psi}_{-}$. $\left|\psi_{-}\right|^{2}-\left|\tilde{\psi}_{-}\right|^{2}$ does not vanish at the minimum of the potential above, but the SUSY is not restored at the local minimum, with a remaining vacuum energy

$$
V=\frac{g_{B}^{2}}{2} \xi_{B}^{2} \frac{g_{C M}^{2}}{g_{C M}^{2}+\left(\cos ^{2} 2 \theta\right) g_{B}^{2}} .
$$

As long as $V(t=0)$ is less than this vacuum energy of the other local minimum, the vacuum we want does not have an instability. Since $V(t=0)$ is sufficiently smaller than the asymptotic value $V(t=\infty)$, there is a good chance that the $V(t=0)$ local minimum is more stable than this new local minimum.

\section{Phenomenology}

We have seen so far that the generalized models in this appendix break both SUSY and $R$-symmetry in the hidden sector, and those breaking are coupled to the messenger fields just like in the field-theory model of section 2. An important difference, however, is that the energy scale of gaugino condensation is not the same as the dynamical scale in the models in this appendix.

Let us take a simple case $N=2$ and $p=1$ as an example. The $\operatorname{SU}(N+p)=$ $\mathrm{SU}(3)$ gauge theory is in the conformal window above the messenger threshold scale [16], and the gauge coupling at the conformal fixed point is roughly $\alpha_{3} \sim(3 \pi / 14)$. Below the messenger scale, the gauge theory with fewer matter fields quickly becomes strongly coupled. The dynamical scale is just below the messenger scale, $\Lambda_{3} \sim M / 4$. We assumed that $32 \pi^{2} \Lambda^{3} / M^{3} \approx \mathcal{O}(1)$ in section 2 in order to avoid negative mass-squared for squarks and sleptons, and this assumption is completely valid.

On the other hand, gaugino condensation in (22) and the meson masses involve an extra parameter $\mu$, whose typical value is at the string scale. To assure the nonperturbative contributions to soft scalar mass squared, we take $\mu \sim \Lambda$. The resulting phenomenology coincides with the one given in the main text.

\footnotetext{
${ }^{11}$ This is essentially due to the charge conjugation symmetry $\mathcal{M}_{12} \leftrightarrow \mathcal{M}_{21}$ discussed in [15. We have assumed that the inclusion of D7-branes does not break the symmetry.
} 


\section{References}

[1] P. J. Fox, A. E. Nelson and N. Weiner, JHEP 0208, 035 (2002) arXiv:hep-ph/0206096.

[2] I. Antoniadis and S. Hohenegger, arXiv:hep-th/0701290.

[3] For a review, G. F. Giudice and R. Rattazzi, Phys. Rept. 322, 419 (1999) arXiv:hep-ph/9801271].

[4] I. R. Klebanov and M. J. Strassler, JHEP 0008, 052 (2000) [arXiv:hep-th/0007191].

[5] A. Dymarsky, I. R. Klebanov and N. Seiberg, JHEP 0601, 155 (2006) arXiv:hep-th/0511254.

[6] K. I. Izawa and T. Yanagida, Prog. Theor. Phys. 114, 433 (2005) arXiv:hep-ph/0501254,

[7] M. Ibe, Y. Nakayama and T. T. Yanagida, arXiv:hep-ph/0703110.

[8] N. Arkani-Hamed and S. Dimopoulos, JHEP 0506, $073 \quad$ (2005) arXiv:hep-th/0405159,

G. F. Giudice and A. Romanino, Nucl. Phys. B 699, 65 (2004) [Erratum-ibid. B 706, 65 (2005)] arXiv:hep-ph/0406088.

[9] N. Arkani-Hamed, M. Dine and S. P. Martin, Phys. Lett. B 431, 329 (1998) arXiv:hep-ph/9803432.

[10] A. E. Nelson and N. Seiberg, Nucl. Phys. B 416, 46 (1994) arXiv:hep-ph/9309299.

[11] S. P. Martin, Phys. Rev. D 55, 3177 (1997) arXiv:hep-ph/9608224.

[12] M. Buican, D. Malyshev, D. R. Morrison, M. Wijnholt and H. Verlinde, JHEP 0701, 107 (2007) arXiv:hep-th/0610007.

[13] A. Butti, M. Grana, R. Minasian, M. Petrini and A. Zaffaroni, JHEP 0503, 069 (2005) arXiv:hep-th/0412187.

[14] P. Ouyang, Nucl. Phys. B 699, 207 (2004) arXiv:hep-th/0311084.

[15] I. R. Klebanov and E. Witten, Nucl. Phys. B 536, 199 (1998) arXiv:hep-th/9807080.

[16] N. Seiberg, Nucl. Phys. B 435, 129 (1995) arXiv:hep-th/9411149.

[17] M. A. Luty, Phys. Rev. D 57 (1998) 1531 arXiv:hep-ph/9706235; A. G. Cohen, D. B. Kaplan and A. E. Nelson, Phys. Lett. B 412 (1997) 301 arXiv:hep-ph/9706275.

[18] E. Poppitz and S. P. Trivedi, Phys. Lett. B 401 (1997) 38 arXiv:hep-ph/9703246. 